International Research Journal of Management, IT \& Social Sciences
Available online at https://sloap.org/journals/index.php/irjmis/
Vol. 6 No. 5, September 2019, pages: 193 203
ISSN: 2395-7492
https://doi.org/10.21744/irjmis.v6n5.732

\title{
Reducing the PDEs to ODEs Through Lie Vectors Using the Integrated Factors
}

\begin{tabular}{|c|c|}
\hline & $\begin{array}{r}\text { Tony Brian Fogel } \\
\text { Steven Segan Lucky } \\
\text { James Arnold Mandy } \\
\text { Berth Bryan Wellbirth }\end{array}$ \\
\hline Article history: & Abstract \\
\hline $\begin{array}{l}\text { Received: } 09 \text { March } 2019 \\
\text { Accepted: } 31 \text { July } 2019 \\
\text { Published: } 03 \text { September } 2019\end{array}$ & $\begin{array}{l}\text { We reduce the PDEs to ODEs through Lie vectors as previously done through } \\
\text { two reduction stages. Some of these ODEs have no solution. Some researchers } \\
\text { in this step, use the SMM, power series method or Riccati equation method to } \\
\text { solve non-solvable equations. We use the integrating factors as a tool to reduce } \\
\text { the order and the nonlinearity in an ODE. This explores new solutions as it } \\
\text { appears for the }(2+1) \text {-dimensional (CBS) and }(3+1) \text {-dimensional generalized } \\
\text { BKP solutions compared results. }\end{array}$ \\
\hline
\end{tabular}

factors;

integrating;

lie;

solutions;

transformation;

2395-7492@ Copyright 2019. The Author. This is an open-access article under the CC BY-SA license (https://creativecommons.org/licenses/by-sa/4.0/) All rights reserved.

\section{Author correspondence:}

Tony Brian Fogel,

Cornell University, Private university in Ithaca, NY 14850, USA.

Email address: fogel.tb@cornell.edu

\section{Introduction}

Recently, many methods are applied to reach analytical solutions of NLPDEs as Darboux transformation (Tang \& Xie, 2011). Moghadam et al., (2013), the $\left(G^{\prime} / G\right)$ expansion method, Exp-Function method and Rational Function transformations (Budd et al., 2006). Here, two equations namely, the (2+1)-dimensional Calogero-BogoyavlenskiiSchiff (CBS) equation and the (3+1)-dimensional generalized BKP equation have been solved by Lie symmetry Reduction method (Ahmad et al., 2015). Using the Lie Reduction method and (IF), we derive a novel combination of solutions for these equations.

a Cornell University, Ithaca, US

${ }^{\mathrm{b}}$ Cornell University, Ithaca, US

c Columbia University, New York, US

${ }^{\mathrm{d}}$ Columbia University, New York, US 


\section{Materials and Methods}

\section{Mathematical Formulation}

In this paragraph, we reduce the nonlinear evolution equations to (ODEs) in three steps. For each used Lie vector, we apply the following steps;

a) The independent variables $(x ; y ; z ; t)$ are reduced to a (PDE) in two variables $(\mathrm{r}, \mathrm{s})$.

b) Evaluate Lie infinitesimals for these PDE then use the evaluated symmetries for a reduction of independent variables from $(r ; s)$ to one variable $(\eta)$.

c) The reduced ODE is non-solvable equations, through their corresponding IF are reduced to new solvable ones.

(2+1)-Dimensional Calogero-Bogoyavlenskii-Schiff equation

$$
u_{x t}+4 u_{x} u_{y x}+2 u_{y} u_{x x}+u_{x x x y}=0
$$

Where $u(x, y, t)$ describes the interaction of Riemann wave propagation in the $x$-direction with the long-wave propagation in the $y$-direction. Tang \& Xie, (2011), derive analytical solutions of (1) using the Tanh-Coth method. Wang et al., (2015), obtained the Noether-type operators associated with the partial Lagrangian for all possible arbitrary functions, followed by a double reduction using symmetries. Li \& Wang (2007), used Lie symmetry with the SMM to present new solutions of CBS equation (1).

Here, we apply two stages of symmetry reduction method to reduce the (CBS) equation to ODEs. During the reduction process, some of the obtained ODEs had no quadrature. We thus solve them using their (IF). Equation (1) has 24 Lie vectors. From the adjoint table, three optimal vectors are deduced.

$$
\begin{aligned}
X_{2} & =\frac{\partial}{\partial t}+\frac{\partial}{\partial u}, \quad X_{7}=t \frac{\partial}{\partial x}+\frac{\partial}{\partial t}+0.5 y \frac{\partial}{\partial u} \\
X_{21} & =\left(1+\frac{1}{4} t x\right) \frac{\partial}{\partial x}+\left(\frac{1}{2} t y\right) \frac{\partial}{\partial y}+\left(\frac{1}{2} t^{2}\right) \frac{\partial}{\partial t}+\left(-\frac{1}{4} t u+\frac{1}{8} x y\right) \frac{\partial}{\partial u}
\end{aligned}
$$

These vectors are utilized to reduce (CBS) (1) three independent variables; $(x ; y ; t)$ to two independent variables; $(r$; $s)$

Reduction of the independent variables in (CBS) equation using $X_{2}$ Lie vector

Equation (1) is transformed through the vector $X_{2}=\frac{\partial}{\partial t}+\frac{\partial}{\partial u}$ to;

$$
4 F_{r} F_{r s}+2 F_{s} F_{r r}+F_{r r s}=0
$$

Where $\mathrm{r}=\mathrm{x}, \mathrm{s}=\mathrm{y}$ and $F(r, s)=u(x, y, t)-t$. The above equation has no exact solution but has six Lie vectors. We choose here to deal only with $\mathrm{V}_{1}, \mathrm{~V}_{5}$ Lie vector as they lead to ODE's having no closed-form solution.

$$
V_{1}=\frac{\partial}{\partial r}+\frac{\partial}{\partial s}, V_{5}=r \frac{\partial}{\partial r}+\frac{\partial}{\partial s}-F \frac{\partial}{\partial F}
$$

Reduction using $V_{1}$

Using $V_{1}(C B S)$ transform to a nonlinear fourth-degree ODE of the following form;

$$
6 \theta_{\eta \eta} \theta_{\eta}-\theta_{\eta \eta \eta \eta}=0
$$


Using Integrating Factor to obtain an exact solution

We first deduce equation (5) IF using maple.

$$
\mu_{1}=\theta_{\eta}, \mu_{2}=1
$$

The IF reduce equation (5) to;

$$
2 \theta_{\eta}{ }^{3}-\theta_{\eta \eta}{ }^{2}=0
$$

This equation has a closed-form solution of the form;

$$
\theta(\eta)=-\frac{2}{\eta+c_{1}}+c_{2}
$$

Where $\eta=-r+s, \theta(\eta)=F(r, s)$ and $c_{1}, c_{2}$ are the integration constants. Back substituting to $(x, y, t)$ for $r=x, s=$ $y, F(r, s)=u(x, y, t)-t$, we get;

$$
u(x, y, t)=-\frac{2}{-x+y+c_{1}}+c_{2}+t
$$

This solution in (9) is plotted in Fig. 1 for two different times; $\mathrm{t}=0, \mathrm{t}=20 \mathrm{sec}$.

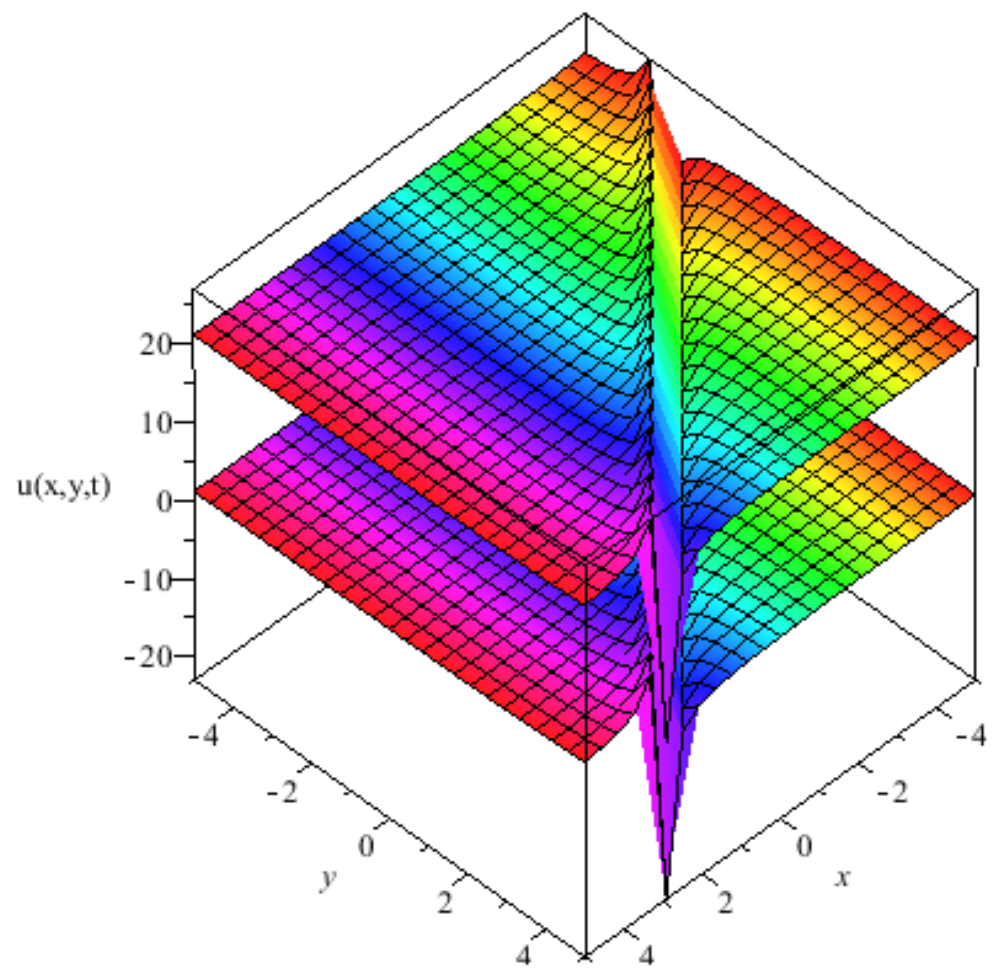

Figure 1. $u(x, y, t)$ at $c_{1}=-2, c_{2}=1, \mathrm{t}=0,20 \mathrm{sec}$.

The wave in Fig. 1 shows a row of peakons moving downward as time passes from zero to twenty.

\section{Reduction using $V_{5}$}

Using the $V_{5},(C B S)$ equation is reduced to a nonlinear ODE of the form;

$$
10 \theta_{\eta}^{2}+6 \theta_{\eta} \theta_{\eta \eta}+8 \theta \theta_{\eta}+4 \theta \theta_{\eta \eta}-6 \theta_{\eta}-11 \theta_{\eta \eta}-6 \theta_{\eta \eta \eta}-\theta_{\eta \eta \eta \eta}=0
$$

The above equation has no exact solution. Using the integrating factor, we can reduce it to a simpler equation.

Fogel, T. B., Lucky, S. S., Mandy, J. A., \& Wellbirth, B. B. (2019). Reducing the PDEs to ODEs through lie vectors using the integrated factors. International Research Journal of Management, IT and Social Sciences, 6(5), 193-203. https://doi.org/10.21744/irjmis.v6n5.732 
We first investigate the IF of (10) using maple.

$$
\mu_{1}=e^{2 \eta}, \mu_{2}=e^{4 \eta} \theta_{\eta}
$$

The IF reduce equation (10) to;

$$
2 \theta_{\eta}{ }^{3}+4 \theta \theta_{\eta \eta}{ }^{2}-3 \theta_{\eta}{ }^{2}-4 \theta_{\eta} \theta_{\eta \eta}-\theta_{\eta \eta}{ }^{2}=0
$$

This equation has an exact solution of the form;

$$
\theta(\eta)=2+c_{1}-\tanh \left(0.5 \eta \sqrt{5+4 c_{1}}+0.5 c_{2} \sqrt{5+4 c_{1}}\right) \sqrt{5+4 c_{1}}
$$

Where $\eta=-\ln (r)+s, \theta(\eta)=r F(r, s)$ and $c_{1}, c_{2}$ are integration constants.

Then back substituting to $(x, y, t)$ where $r=x, s=y, F(r, s)=u(x, y, t)-t$, we obtain;

$$
u(x, y, t)=\frac{2+c_{1}-\tanh \left(0.5(-\ln (x)+y) \sqrt{5+4 c_{1}}+0.5 c_{2} \sqrt{5+4 c_{1}}\right) \sqrt{5+4 c_{1}}+t}{x}
$$

This solution is plotted in Fig. 2 in a complex domain. The peakon waves depicted in Fig. 2 decay with time.

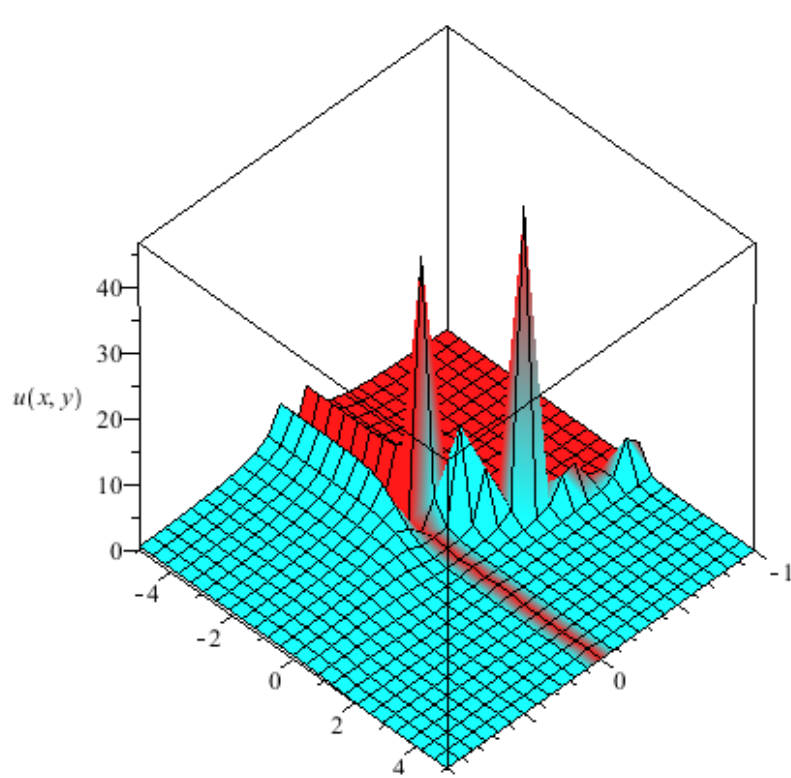

(a)

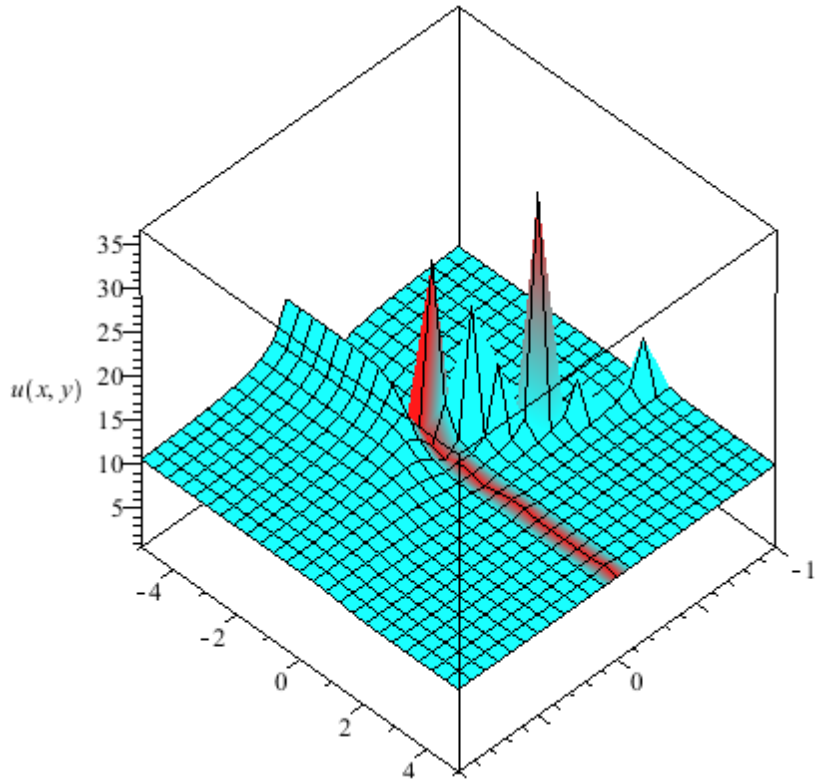

(b)

Figure 2. (a) $u(x, y, t)$ at $c_{1}=1, c_{2}=1, \mathrm{t}=0 \mathrm{sec}$ (b) $u(x, y, t)$ at $c_{1}=1, c_{2}=1, \mathrm{t}=10 \mathrm{sec}$.

By comparison of the results obtained, our solution is new

Reduction of the independent variables in (CBS) equation using $X_{7}$ Lie vector

Equation (1) is transformed through the optimal vector $\boldsymbol{X}_{\mathbf{7}}$ to

$$
16 F_{S} F_{r s}+8 F_{r}\left(F_{S S}\right)-8 F_{s s r}=0
$$

Where $r=y, s=-2 x+t^{2}, F(r, s)=u(x, y, t)-0.5 t y$,

This equation hasn't an exact solution but has a six Lie vectors. We choose to work only with $\mathrm{V}_{1}$. This Lie vector leads to an ODE with no analytic solution. While the rest of the vector lead to solvable ODEs. 


$$
V_{1}=\frac{\partial}{\partial r}+\frac{\partial}{\partial s}
$$

Using $V_{1}$ transform $(C B S)$ to a nonlinear fourth-degree ODE of the following form;

$$
3 \theta_{\eta \eta} \theta_{\eta}-\theta_{\eta \eta \eta \eta}=0
$$

Using Integrating Factor to obtain a closed-form solution

We first investigate the IF of (17) using maple.

$$
\mu_{1}=\theta_{\eta}, \mu_{2}=1
$$

We use the two-integrating factor to reduce equation (17) to;

$$
\theta_{\eta}^{3}-\theta_{\eta \eta}{ }^{2}=0
$$

This equation has an explicit solution in the form;

$$
\theta(\eta)=-\frac{4}{\eta+c_{1}}+c_{2}
$$

where $\eta=-r+s, \theta(\eta)=F(r, s)$ and $c_{1}, c_{2}$ are integration constants.

Then back to $(x, y, t)$ coordinates where $r=y, s=-2 x+t^{2}, F(r, s)=u(x, y, t)-0.5 t y$, we obtain;

$$
u(x, y, t)=-\frac{4}{-y-2 x+t^{2}+c_{1}}+c_{2}+0.5 t y
$$

This solution is plotted in Fig. 3. The wave peak position changes with time.
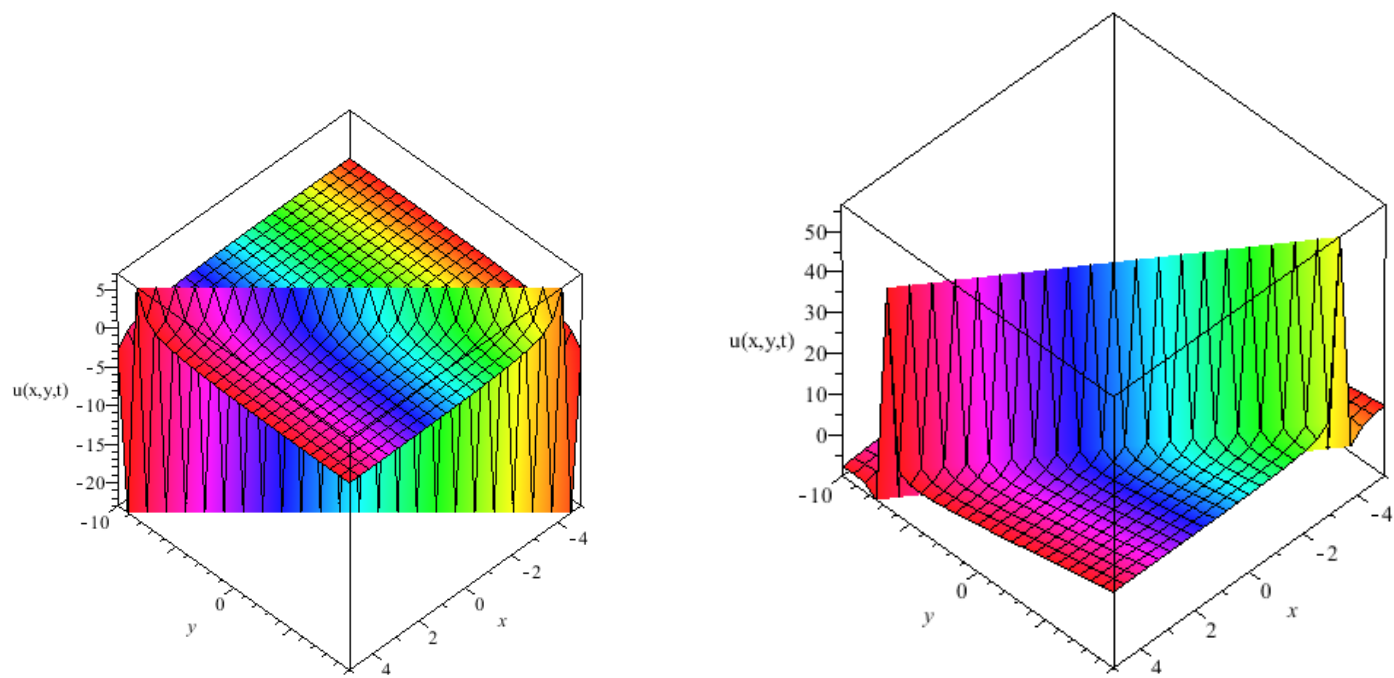

Figure 3. $u(x, y, t)$ at $c_{1}=1, c_{2}=1, \mathrm{t}=0,1.5 \mathrm{sec}$.

Reduction of the independent variables in (CBS) equation using $X_{21}$ Lie vector

Equation (1) is transformed through the vector $\boldsymbol{X}_{\mathbf{2 1}}$ to;

$$
8 F_{r}^{2}+2 F F_{S S}+4 F_{S S S}+2 r F_{S} F_{r s}+4 r F_{r}\left(F_{S S}\right)+s F_{S S S S}=0
$$

The reduced equation (22) has no closed-form solution but have six Lie vectors. We choose to work with $\mathrm{V}_{2}$, as follow;

$$
V_{2}=r \frac{\partial}{\partial r}+\left(\frac{1}{2} s+\sqrt{r}\right) \frac{\partial}{\partial s}-\frac{1}{2} F \frac{\partial}{\partial F}
$$

Using $V_{2}$ transform (CBS) to a nonlinear fourth degree ODE in the following form;

Fogel, T. B., Lucky, S. S., Mandy, J. A., \& Wellbirth, B. B. (2019). Reducing the PDEs to ODEs through lie vectors using the integrated factors. International Research Journal of Management, IT and Social Sciences, 6(5), 193-203. 


$$
6 \theta_{\eta \eta} \theta_{\eta}+\theta_{\eta \eta \eta \eta}=0
$$

\section{Using Integrating Factor to obtain an exact solution}

We get IF for (24) using maple.

$$
\mu_{1}=\theta_{\eta}, \mu_{2}=1
$$

The IF reduce equation (24) to;

$$
2 \theta_{\eta}^{3}+\theta_{\eta \eta}^{2}=0
$$

This equation has a closed-form solution;

$$
\theta(\eta)=\frac{2}{\eta+c_{1}}+c_{2}
$$

where $\eta=\frac{s-\sqrt{r} \ln (r)}{\sqrt{r}}, \theta(\eta)=\sqrt{r} F(r, s)$ and $c_{1}, c_{2}$ are integration constants.

Then back substituting to $(\mathrm{x}, \mathrm{y}, \mathrm{t})$ where $r=\frac{t}{y}, s=\frac{3 t x+4}{3 \sqrt{y} t}, F(r, s)=-\left(-u(x, y, t)+\frac{y x}{4 t}+\frac{y}{t^{2}}\right)$ we obtain;

$$
u(x, y, t)=\frac{2}{\frac{3 t x+4}{3 t}-\sqrt{t} \ln \left(\frac{t}{y}\right)+c_{1}}+c_{2}\left[\frac{3 t x+4}{3 \sqrt{y} t}-\sqrt{\frac{t}{y}} \ln \left(\frac{t}{y}\right)+c_{1}\right]+\frac{y}{t^{2}}+\frac{y x}{4 t}
$$

This solution is plotted in Fig. 4.

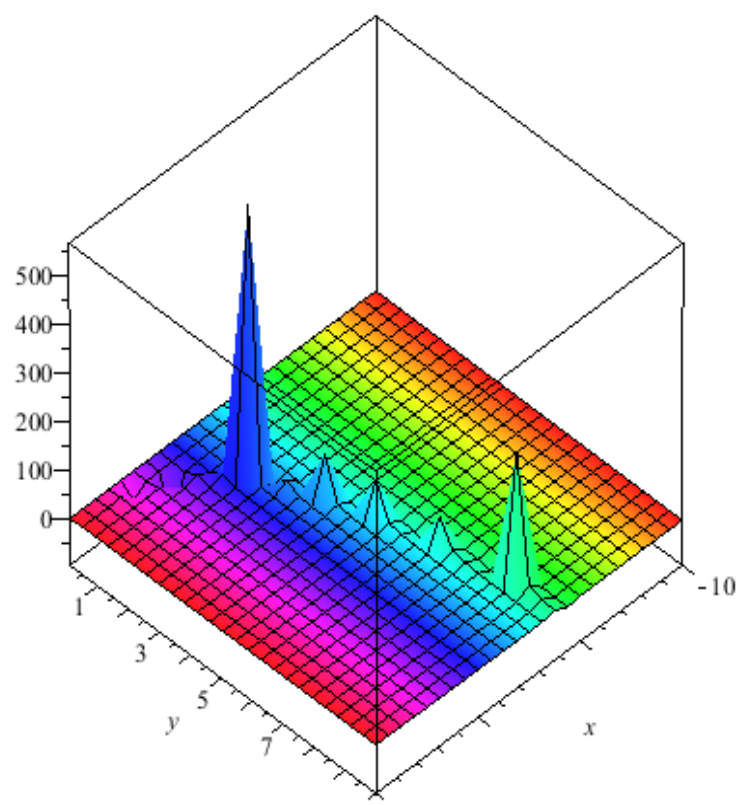

(a)

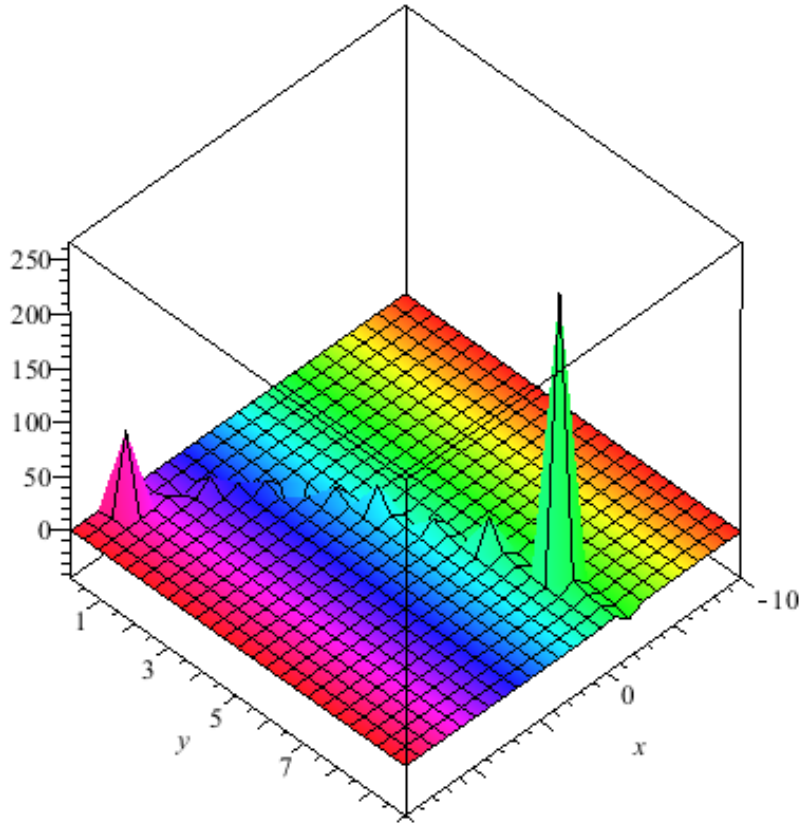

(b)

Figure 4. (a) $u(x, y, t)$ at $c_{1}=1, c_{2}=1, \mathrm{t}=5 \mathrm{sec}$. (b) $u(x, y, t)$ at $c_{1}=1, c_{2}=1, \mathrm{t}=20 \mathrm{sec}$.

The peakon waves move on a parabolic axis drifting to the right with time. This result is new. 


\section{Results and Discussions}

\section{(3+1)-Dimensional B-Kadomtsev-Petviashvili (BKP) Equation}

Susto \& Krstic (2010), use Lie symmetry analysis, Riccati equation and power series method to introduce specific solutions for (3+1) BKP equation. Also, Hasan et al., (2016), apply prorogation theorem to get the similarity variables and use these generators to solve the equation. In this paper, we consider the BKP equation in $(3+1)$ dimensional space as;

$$
u_{y t}-u_{x x x y}-3 u_{y} u_{x x}-3 u_{x} u_{x y}+6 u_{x x}-3 u_{z z}=0
$$

This equation has 36 Lie vectors, and we will choose $X_{13}$ to reduce the equation;

$$
X_{13}=\frac{\partial}{\partial z}+t z \frac{\partial}{\partial u}, X_{1}+X_{9}+X_{13}=\frac{\partial}{\partial x}+\frac{\partial}{\partial y}+\frac{\partial}{\partial Z}+t z \frac{\partial}{\partial u}
$$

The PDE (29) function of four independent variables; $(x ; y ; t ; z)$ is first reduced to a PDE in three independent variables, $(l ; h ; o)$, using its Lie vectors (30) then reduce to two independent variables $(r ; s)$ and then one independent $\eta$.

Using $X_{13}$ Lie Vector to reduce the Independent Variables in (BKP) Equation

Equation (29) is transformed through the vector $X_{13}=\frac{\partial}{\partial z}+t z \frac{\partial}{\partial u}$ to;

$$
K_{o h}-\left(K_{l l l h}\right)-3 K_{l l} K_{h}-3 K_{l h} K_{l}+6 K_{l l}-3 o=0
$$

This equation has no closed form solution, but possesses 12 Lie vectors; we choose $\mathrm{V}_{1}$;

$$
V_{1}=\frac{\partial}{\partial l}+\frac{\partial}{\partial h}
$$

This vector transforms the equation (31) to;

$$
F_{r s}-6 F_{r}\left(F_{r r}\right)+6 F_{r r}+F_{r r r r}-3 s=0
$$

This equation has no closed-form solution, but have eight Lie vectors; we will choose here to work only with $\mathrm{e}_{5}$

$$
e_{5}=\left(\frac{1}{3} r-8 s^{3}\right) \frac{\partial}{\partial r}+s \frac{\partial}{\partial s}+\left(1+4 r s^{2}+\frac{2}{3} r-\frac{1}{3} F\right) \frac{\partial}{\partial F}
$$

Using $e_{5}$, transform (33) to the following form;

$$
-54 \theta_{\eta \eta}-\theta_{\eta \eta \eta \eta}+18 \theta_{\eta \eta} \theta_{\eta}=0
$$

This is ODE has no analytical solution.

Using the Integrating Factor to obtain an explicit solution

We first deduce equation (35) IF using maple.

$$
\mu_{1}=\theta_{\eta}, \mu_{2}=1
$$

The IF reduce equation (35) to;

$$
\theta_{\eta \eta}{ }^{2}-6 \theta_{\eta}^{3}+54 \theta_{\eta}^{2}=0
$$

This equation has a closed-form solution of the form;

$$
\theta(\eta)=\sqrt{6} \tan \left(\frac{3 \sqrt{6}}{2} \eta+\frac{3 \sqrt{6}}{2} c_{1}\right)-\sqrt{6} \tan ^{-1}\left(\tan \left(\frac{3 \sqrt{6}}{2} \eta+\frac{3 \sqrt{6}}{2} c_{1}\right)\right)+9 \eta+c_{2}
$$

Fogel, T. B., Lucky, S. S., Mandy, J. A., \& Wellbirth, B. B. (2019). Reducing the PDEs to ODEs through lie vectors using the integrated factors. International Research Journal of Management, IT and Social Sciences, 6(5), 193-203. 
where $\eta=\frac{1}{3} r+s^{3}, \theta(\eta)=F(r, s)=/ s+\frac{27^{\frac{2}{3}}}{5} s^{5}-\frac{27^{\frac{2}{3}}}{6} s^{2} r-\frac{27^{\frac{2}{3}}}{2} s^{5}$.

Then back to (l; h;o) with $r=-l+h, s=o, F(r, s)=K(l, h, o) ; h=y, l=x, o=t, K(l, h, o)=$ $u(x, y, t, z)-0.5 z^{2}$ we obtain;

$$
\begin{aligned}
u(x, y, t, z)=\sqrt{6} & \tan \left(\frac{3 \sqrt{6}}{2}\left(\frac{1}{3}(-x+y)+t^{3}\right)+\frac{3 \sqrt{6}}{2} c_{1}\right)-\sqrt{6} \tan ^{-1}\left(\tan \left(\frac{3 \sqrt{6}}{2}\left(\frac{1}{3}(-x+y)+t^{3}\right)+\frac{3 \sqrt{6}}{2} c_{1}\right)\right) \\
+9 & \left(\frac{1}{3}(-x+y)+t^{3}\right)+c_{2}+0.5 t z^{2}+t+\frac{27}{10} t^{5}+\frac{3}{2} t^{2}(-\mathrm{x}+\mathrm{y})
\end{aligned}
$$

This solution is plotted in Fig. 5 for different values of times. It shows a change in the peak wave position as time increases and by increasing the time the surface of the wave follows a parabolic path whose amplitude decreases with time.

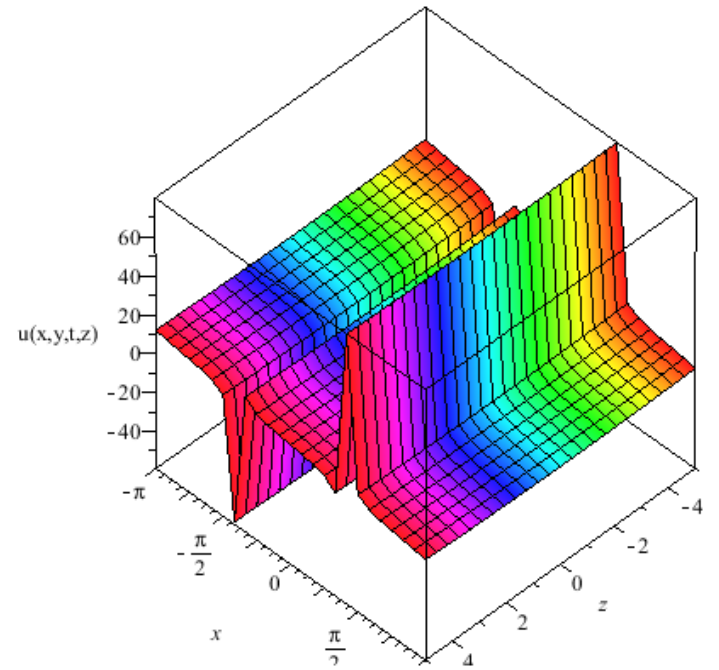

Figure 5. (a) $u(x, y, t, z)$ at $c_{1}=0, c_{2}=1$, $\mathrm{y}=0, \mathrm{t}=0.1 \mathrm{sec}$.

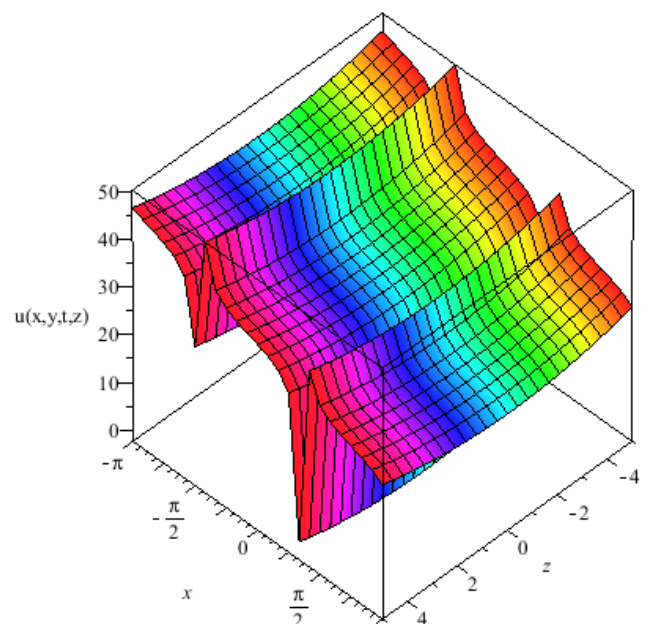

(b) $u(x, y, t, z)$ at $c_{1}=0, c_{2}=1$, $\mathrm{y}=0, \mathrm{t}=0.3 \mathrm{sec}$

Using $X_{1}+X_{9}+X_{13}$ Lie Vector to reduce the Independent Variables in (BKP) Equation

Equation (29) is transformed through the vector $X_{1}+X_{9}+X_{13}=\frac{\partial}{\partial x}+\frac{\partial}{\partial y}+\frac{\partial}{\partial z}+t z \frac{\partial}{\partial u}$ to;

$K_{l h}+K_{l l l l}+3 K_{o l l l}+3 K_{o o l l}+\left(K_{o o o l}\right)-6 K_{l} K_{l l}-9 K_{l o} K_{l}-3 K_{l} K_{o o}+3 h K_{l}-3 K_{l l} K_{o}-3 K_{l o} K_{o}+12 K_{l o}$

$$
+3 o h K_{l l}+3 o h K_{l o}+6 K_{l l}+3 K_{o o}-3 h=0
$$

This equation has no closed-form solution, but possesses 12 Lie vectors; we choose $\mathrm{V}_{1}$;

$$
V_{1}=2 \frac{\partial}{\partial l}+\frac{\partial}{\partial o}+h o \frac{\partial}{\partial K}
$$

Equation (40) is transformed to;

$$
0.5 s-\frac{3}{4} s r F_{s s}-\frac{1}{16} F_{s s s s}-0.5 F_{r s}-\frac{3}{4} r F_{s}+\frac{3}{4} F_{s} F_{s s}-\frac{3}{2} F_{s s}+\frac{3}{4} s r^{2}-\frac{3}{2} r=0
$$

This equation has no closed-form solution, but has eight Lie vectors; we choose to work only with $\mathrm{e}_{5}$

$$
e_{5}=\frac{\partial}{\partial r}+\frac{3}{2} r^{2} \frac{\partial}{\partial s}+\left(1+0.5 s^{2}+\frac{9}{2} r^{3} s-6 r s\right) \frac{\partial}{\partial F}
$$

As this Lie vector leads to an ODE with no analytic solution. Using $e_{5}$, transform (BKP) to a nonlinear fourth degree ODE of the form;

$$
-24 \theta_{\eta \eta}-\theta_{\eta \eta \eta \eta}+12 \theta_{\eta \eta} \theta_{\eta}=0
$$


Using Integrating Factor to get an explicit solution

We first deduce equation (44) IF using maple.

$$
\mu_{1}=\theta_{\eta}, \mu_{2}=1
$$

The integrating factor $\mu_{1}$ reduces equation (44) to;

$$
\theta_{\eta \eta}{ }^{2}-4 \theta_{\eta}^{3}+24 \theta_{\eta}^{2}=0
$$

This equation has a closed-form solution;

$$
\theta(\eta)=\sqrt{6} \tan \left(\sqrt{6} \eta+\sqrt{6} c_{1}\right)-\sqrt{6} \tan ^{-1}\left(\tan \left(\sqrt{6} \eta+\sqrt{6} c_{1}\right)\right)+6 \eta+c_{2}
$$

where $\eta=-\frac{3}{2} r^{3}+s, \theta(\eta)=F(r, s)-s+\frac{27}{10} r^{5}+3 r^{2} s-\left(1+\frac{1}{2} s^{2}\right) \mathrm{r}$.

Then back to $(l ; h ; o)$ coordinates with

That leads to;

$$
\begin{gathered}
r=h, s=\frac{-l}{2}+o, F(r, s)=K(l, h, o)+\frac{1}{8} h l^{2}-\frac{h l o}{2} ; h=t, \\
l=-x+y, o=-x+z, \quad K(l, h, o)=u(x, y, t, z)+0.5 x^{2} t-x t z
\end{gathered}
$$

$$
\begin{aligned}
u(x, y, t, z)=\sqrt{6} & \tan \left(\sqrt{6}\left(-\frac{3}{2} t^{3}-\frac{x+y}{2}+z\right)+\sqrt{6} c_{1}\right)-\sqrt{6} \tan ^{-1}\left(\tan \left(\sqrt{6}\left(-\frac{3}{2} t^{3}-\frac{x+y}{2}+z\right)+\sqrt{6} c_{1}\right)\right) \\
+ & \left(1+0.5\left(\frac{-\mathrm{x}-\mathrm{y}}{2}+z\right)^{2}\right)+c_{2}-9 t^{3}-3 x-3 y+6 z+\frac{27}{10} t^{5}-3 t^{2}\left(\frac{-\mathrm{x}-\mathrm{y}}{2}+z\right) \\
& -0.5 x^{2} t+x t z-\frac{t}{8}(-x+y)^{2}+\frac{t(-x+y)(-x+z)}{2}+\left(1+\frac{1}{2}\left(\frac{-x-y}{2}+z\right)^{2}\right)
\end{aligned}
$$

This wave is plotted in Fig. 6. A series of solitons inverse its flow at $t=0.1 \mathrm{sec}$.

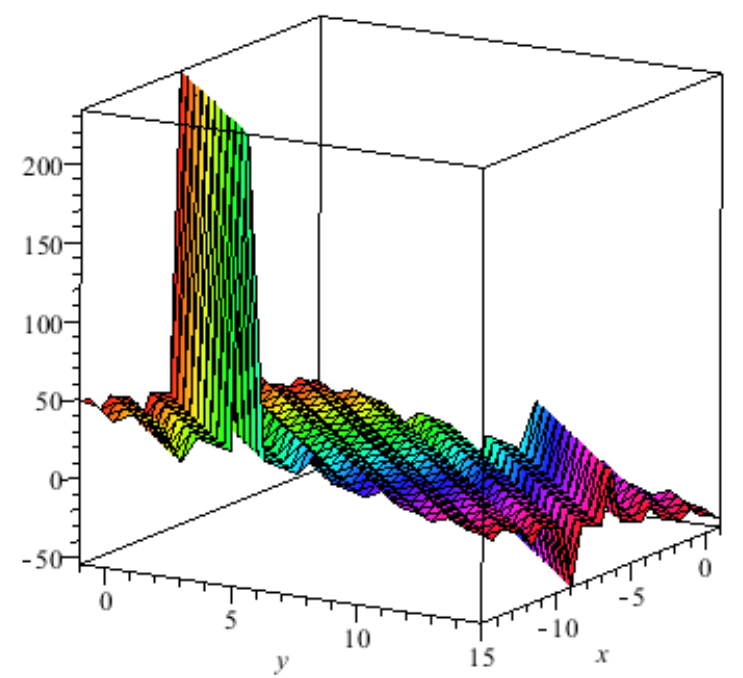

(a)

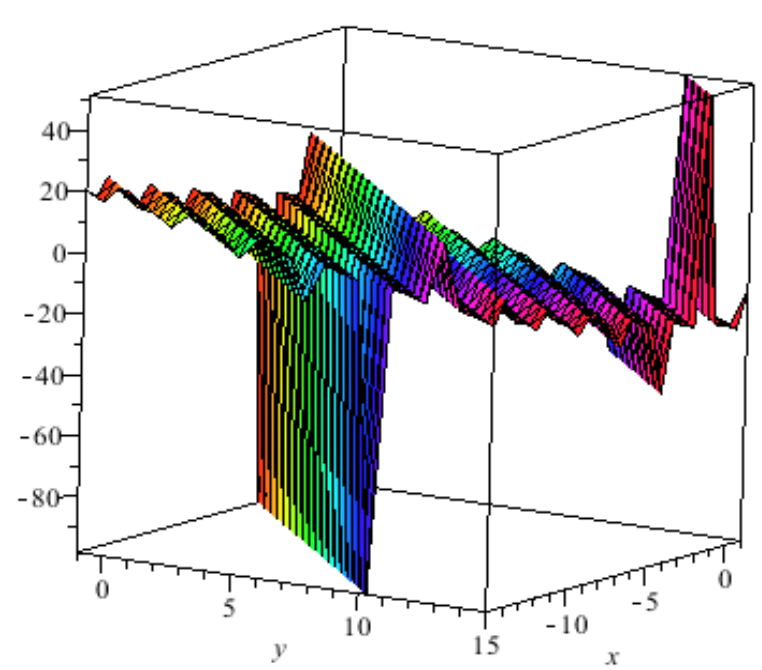

(b)

Figure 6. (a) $\mathrm{u}(\mathrm{x}, \mathrm{y}, \mathrm{t}, \mathrm{z})$ at $c_{1}=1, c_{2}=1, \mathrm{z}=0.1$ at time $\left.\mathrm{t}=0 ; \quad \mathrm{b}\right) \mathrm{u}(\mathrm{x}, \mathrm{y}, \mathrm{t}, \mathrm{z})$ at $c_{1}=1, c_{2}=1, \mathrm{z}=0.1$ at time $\mathrm{t}=1$ sec.

The groups of soliton waves are decay with time and run towards left. Here, we compare our result in (48) with two solutions in (Krstic, 2009; Ren et al., 2013), we find that;

Fogel, T. B., Lucky, S. S., Mandy, J. A., \& Wellbirth, B. B. (2019). Reducing the PDEs to ODEs through lie vectors using the integrated factors. International Research Journal of Management, IT and Social Sciences, 6(5), 193-203. 
a) Simsen \& Simsen (2011), used some of the generators and obtained some traveling wave solution. His solutions in most cases, localized only in three dependent variables $(\mathrm{x}, \mathrm{y}, \mathrm{t})$. We plot his result presented by $\mathrm{X}_{4}$ in Fig. 7;

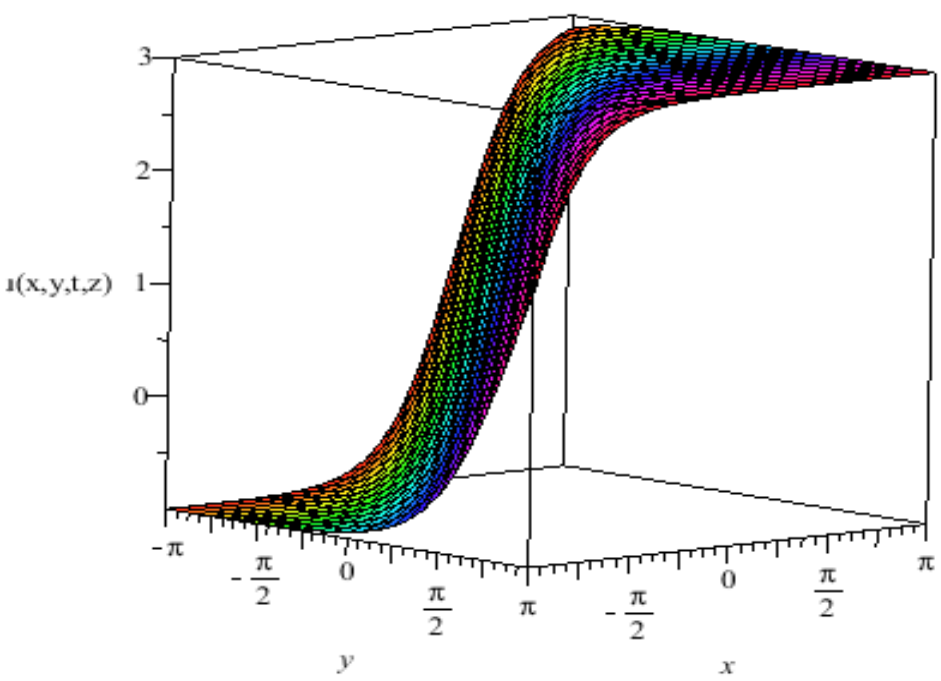

Figure 7. $u(x, y, t, z)=2 c_{2} \tanh \left(1 / 4\left(\frac{4 c_{2} c_{2} x+4 c_{2} c_{4} t+4 c_{1} c_{2}+3 y}{c_{2}}\right)\right)+c_{5}$ for $c_{1}=1, c_{2}=c_{4}=c_{5}=$ at time $\mathrm{t}=0$

b) In Fig 7, he presented the travelling wave solution while we found a multi peaks solution as depicted in Fig. 6.

The solution in equation (23) presented in Zgliczynski (2003), in three variables $(x, y, t)$ is different from our solution.

\section{Conclusion}

Here, we reduce the PDEs to ODEs through Lie vectors as previously done in Bridges \& Reich (2001), through two reduction stages. Some of these ODEs have no solution. Some researchers in this step, use the SMM, power series method or Riccati equation method to solve non-solvable equations. We use the integrating factors as a tool to reduce the order and the nonlinearity in an ODE. This explores new solutions as it appears for the (2+1)-dimensional (CBS) and (3+1)-dimensional generalized BKP solutions compared with Arcak (2011) results.

\section{Conflict of interest statement}

The authors declared that they have no competing interest.

\section{Statement of authorship}

The authors have a responsibility for the conception and design of the study. The authors have approved the final article.

\section{Acknowledgments}

The authors would like to thank the editor of IRJMIS for their valuable time, support, and advice in completing the present research. 


\section{References}

Ahmad, F., Tohidi, E., Ullah, M. Z., \& Carrasco, J. A. (2015). Higher order multi-step Jarratt-like method for solving systems of nonlinear equations: Application to PDEs and ODEs. Computers \& Mathematics with Applications, 70(4), 624-636. https://doi.org/10.1016/j.camwa.2015.05.012

Arcak, M. (2011). Certifying spatially uniform behavior in reaction-diffusion PDE and compartmental ODE systems. Automatica, 47(6), 1219-1229. https://doi.org/10.1016/j.automatica.2011.01.010

Bridges, T. J., \& Reich, S. (2001). Multi-symplectic integrators: numerical schemes for Hamiltonian PDEs that conserve symplecticity. Physics Letters A, 284(4-5), 184-193. https://doi.org/10.1016/S0375-9601(01)00294-8

Budd, C., Koch, O., \& Weinmüller, E. (2006). From nonlinear PDEs to singular ODEs. Applied Numerical Mathematics, 56(3-4), 413-422. https://doi.org/10.1016/j.apnum.2005.04.012

Hasan, A., Aamo, O. M., \& Krstic, M. (2016). Boundary observer design for hyperbolic PDE-ODE cascade systems. Automatica, 68, 75-86. https://doi.org/10.1016/j.automatica.2016.01.058

Krstic, M. (2009). Compensating actuator and sensor dynamics governed by diffusion PDEs. Systems \& Control Letters, 58(5), 372-377. https://doi.org/10.1016/j.sysconle.2009.01.006

Li, X., \& Wang, M. (2007). A sub-ODE method for finding exact solutions of a generalized KdV-mKdV equation with high-order nonlinear terms. Physics Letters A, 361(1-2), 115-118.

Moghadam, A. A., Aksikas, I., Dubljevic, S., \& Forbes, J. F. (2013). Boundary optimal (LQ) control of coupled hyperbolic PDEs and ODEs. Automatica, 49(2), 526-533. https://doi.org/10.1016/j.automatica.2012.11.016

Ren, B., Wang, J. M., \& Krstic, M. (2013). Stabilization of an ODE-Schrödinger cascade. Systems \& Control Letters, 62(6), 503-510. https://doi.org/10.1016/j.sysconle.2013.03.003

Simsen, J., \& Simsen, M. S. (2011). PDE and ODE limit problems for p (x)-Laplacian parabolic equations. Journal of Mathematical Analysis and Applications, 383(1), 71-81. https://doi.org/10.1016/j.jmaa.2011.05.003

Susto, G. A., \& Krstic, M. (2010). Control of PDE-ODE cascades with Neumann interconnections. Journal of the Franklin Institute, 347(1), 284-314. https://doi.org/10.1016/j.jfranklin.2009.09.005

Tang, S., \& Xie, C. (2011). Stabilization for a coupled PDE-ODE control system. Journal of the Franklin Institute, 348(8), 2142-2155. https://doi.org/10.1016/j.jfranklin.2011.06.008

Tang, S., \& Xie, C. (2011). State and output feedback boundary control for a coupled PDE-ODE system. Systems \& Control Letters, 60(8), 540-545. https://doi.org/10.1016/j.sysconle.2011.04.011

Wang, J. M., Liu, J. J., Ren, B., \& Chen, J. (2015). Sliding mode control to stabilization of cascaded heat PDE-ODE systems subject to boundary control matched disturbance. Automatica, 52, $23-34$. https://doi.org/10.1016/j.automatica.2014.10.117

Zgliczynski, P. (2003). On smooth dependence on initial conditions for dissipative PDEs, an ODE-type approach. Journal of Differential Equations, 195(2), 271-283. https://doi.org/10.1016/j.jde.2003.07.009

Fogel, T. B., Lucky, S. S., Mandy, J. A., \& Wellbirth, B. B. (2019). Reducing the PDEs to ODEs through lie vectors using the integrated factors. International Research Journal of Management, IT and Social Sciences, 6(5), 193-203. https://doi.org/10.21744/irjmis.v6n5.732 